\title{
Integrität in der Wissenschaft: eine zwingende Voraussetzung für das Vertrauen der Öffentlichkeit
}

Die Förderung von Kreativität, Originalität der Ideen und Qualität der Forschungsprogramme ist eine der Grundlagen der wissenschaftlichen Gemeinschaft. Das System der Evaluation von Projekten und Publikationen durch Fachkollegen und unabhängige Experten («peer review») ist breit akzeptiert. Im Gegensatz dazu fehlt ein standardisiertes Vorgehen zur Beurteilung von Forschungsarbeiten und Wissenschaftlern, bei denen der Verdacht besteht, dass sie die Regeln der Ethik und der intellektuellen Redlichkeit nicht strikte befolgen. Auch wenn solche Fälle glücklicherweise selten sind, erscheint ein systematisches und objektives Abklärungsverfahren notwendig.

Im Sommer 1999 setzte die Schweizerische Akademie der Medizinischen Wissenschaften (SAMW) eine «Kommission für die wissenschaftliche Integrität in der medizinischen und biomedizinischen Forschung» ein. Diese legt nun Richtlinien vor, welche genau und sorgfältig die Verhaltensregeln für Forscher und Gutachter definieren (diese sind in die Ausbildung von jungen Forschern zu integrieren) und das Vorgehen bei Verdacht auf Nichtbeachtung der Regeln bzw. Betrug skizzieren. Darüber hinaus weist der Text den Institutionen eine wichtige Funktion zu, wenn es darum geht, Hinweise auf
Betrug abzuklären - dies durch die Etablierung einer «Integritätsschutzorganisation». Der Senat der SAMW hat an seiner Sitzung vom Mai 2002 die Richtlinien verabschiedet und die Medizinischen und Veterinärmedizinischen Fakultäten sowie die FMH eingeladen, diese zu «ratifizieren», d.h. für ihre Mitglieder verbindlich zu erklären (diesem Ansinnen sind mehrere der angeschriebenen Institutionen bereits nachgekommen).

Das Bild der Wissenschaft mit all ihren Entwicklungen und mit dem Fortschritt, den sie tagtäglich für das Verständnis des Lebens und der Umwelt mit sich bringt, ist zwingend auf eine lückenlose Ehrlichkeit angewiesen. Die Integrität des Wissenschaftlers und seiner Arbeit trägt zu einem besseren Verständnis, zu grösserer Anerkennung und stärkerem Vertrauen von seiten der Gesellschaft bei.

Die SAMW hofft, dass diese Richtlinien auch andere Forschungsinstitutionen anregen werden, sich mit diesen Fragen zu beschäftigen, um so die Glaubwürdigkeit der Forschenden und ihrer Aktivitäten zu erhöhen.

Prof. Peter Suter Vizepräsident der SAMW und Dekan der Medizinischen Fakultät Genf 


\section{Intégrité dans la science - une condition indispensable pour la confiance du public}

La promotion de la créativité, de l'originalité des idées et de la qualité des programmes de recherche est un des principes de base du monde scientifique. Pour l'évaluation des projets, puis des publications, le système des «pairs» et experts indépendants est dès lors bien établi. Par contre l'évaluation des travaux et des chercheurs ne respectant pas strictement les règles d'éthique et d'honnêteté intellectuelle n'est pas encore bien codifiée. Même s'il s'agit de faits heureusement très rares, une approche très systématique et objective est nécessaire.

Au cours de l'été 1999, l'Académie Suisse des Sciences Médicales (ASSM) a mis sur pied une «commission pour l'intégrité scientifique dans le domaine de la recherche médicale et biomédicale», qui propose maintenant des directives très précises, d'une part pour définir soigneusement les règles de comportement des chercheurs et des experts (à intégrer dans la formation des jeunes scientifiques), et d'autre part pour donner un cadre approprié à l'investigation de suspicions ou d'accusations de non respect de ces règles ou tout simplement de fraude. En outre, ce texte définit le rôle des institutions dans l'analyse de dénonciations pour fraude, par la création d'une organisation de défense de l'intégrité. Le sénat de l'ASSM, lors de sa séance de mai 2002, a approuvé ces directives et a invité les facultés de médecine et la FMH à les ratifier, c'est-à-dire qu'elles engagent leurs membres (plusieurs institutions contactées ont déjà répondu favorablement à cette demande).

L'image de la science et des développements et progrès qu'elle apporte tous les jours à la compréhension de la vie et du monde qui nous entoure nécessite impérativement une honnêteté sans faille. Ainsi, l'intégrité du scientifique et de son travail va servir à une meilleure compréhension, une reconnaissance et une confiance accrues de la part du grand public.

$\mathrm{Au}$ travers de telles directives, L'Académie Suisse des Sciences Médicales souhaite inviter les autres organisations de recherche à se préoccuper aussi de ces questions, et définir un cadre pour les scientifiques en garantissant ainsi la crédibilité de leurs activités.

Prof. Peter Suter Vice-président de l'ASSM et Doyen da la Faculté de médecine de Genève 